\title{
Mortalidade por câncer de mama em mulheres de Santa Catarina, Brasil, 2000-2017
}

\author{
Mortality due to breast cancer in women of Santa Catarina, Brazil, 2000-2017 \\ Mortalidad por cáncer de mama en mujeres de Santa Catarina, Brasil, 2000-2017
}

Recebido: 06/10/2021 | Revisado: 12/10/2021 | Aceito: 19/10/2021 | Publicado: 20/10/2021

\author{
Maria Isabel Gonçalves da Silva \\ ORCID: https://orcid.org/0000-0001-7768-7531 \\ Universidade Comunitária da Região de Chapecó, Brasil \\ E-mail: maisabel@unochapeco.edu.br \\ Jane Kelly Oliveira Friestino \\ ORCID: https://orcid.org/0000-0002-5432-9560 \\ Universidade Federal da Fronteira Sul, Brasi \\ E-mail: jane.friestino@uffs.edu.br \\ Priscila Maria Stolses Bergamo Francisco \\ ORCID: https://orcid.org/0000-0001-7361-9961 \\ Universidade Estadual de Campinas, Brasil \\ E-mail: primaria@unicamp.br \\ Marcelo Moreno \\ ORCID: https://orcid.org/0000-0003-0244-9138 \\ Universidade Federal da Fronteira Sul, Brasil \\ E-mail: marcelo.moreno@uffs.edu.br \\ Vanessa da Silva Corralo \\ ORCID: https://orcid.org/0000-0003-4234-4875 \\ Universidade Comunitária da Região de Chapecó, Brasil \\ E-mail: vcorralo@unochapeco.edu.br
}

\begin{abstract}
Resumo
Objetivou-se neste estudo analisar a tendência dos coeficientes de mortalidade por câncer de mama em mulheres no estado de Santa Catarina, Brasil, entre os anos de 2000 e 2017. Trata-se de um estudo ecológico realizado com dados de óbitos do Sistema de Informações sobre Mortalidade e dados populacionais obtidos das estimativas disponibilizadas pelo Ministério da Saúde. Na análise foram usados modelos de regressão linear simples e polinomial. Entre os anos de 2000 e 2017, foram registrados 7.363 óbitos por câncer de mama em mulheres com idade igual ou maior a 20 anos. Para o período como um todo, $47,5 \%$ do total de óbitos ocorreu na faixa etária de 50 a 69 anos $(\mathrm{n}=3.497)$. O coeficiente médio de mortalidade por câncer de mama foi de 18,24/100.000 mulheres (2000-2017). Os coeficientes médios de mortalidade superaram 30 óbitos/100.000 mulheres na faixa etária de 50 a 59 anos, chegando a 87,25/100.000 nas mais longevas. Na análise da tendência desagregando-se por faixas etárias, observou-se em geral, incremento linear não constante no período. O estudo revelou uma tendência crescente de mortalidade por câncer de mama em mulheres residentes no estado de Santa Catarina no período estudado, e mostrou a magnitude do aumento dos coeficientes médios de acordo com as faixas etárias consideradas.

Palavras-chave: Aumento da mortalidade; Epidemiologia; Estudos de séries temporais; Neoplasias da mama; Saúde da mulher.

Abstract

The aim of this study was to analyze the trend of breast cancer mortality rates in women in the state of Santa Catarina, Brazil, between 2000 and 2017. This is an ecological study carried out with death data from the Information System on Mortality and population data obtained from estimates made available by the Ministry of Health. Simple linear and polynomial regression models were used in the analysis. Between 2000 and 2017, 7,363 deaths from breast cancer were recorded in women aged 20 years or over. For the period, $47.5 \%$ of the total deaths occurred in the age group from 50 to 69 years $(n=3,497)$. The average breast cancer mortality rate was $18.24 / 100,000$ women $(2000-2017)$. The average mortality coefficients exceeded 30 deaths/100,000 women aged 50 to 59 years, reaching 87.25/100,000 in the longest-lived. In the analysis of the trend, broken down by age groups, it was observed, in general, a non-constant linear increase in the period. The study revealed an increasing trend of mortality from breast cancer in women living in the state of Santa Catarina during the period and showed the magnitude of the increase in the mean coefficients according to the age groups considered.
\end{abstract}

Keywords: Increased mortality; Epidemiology; Time series studies; Breast neoplasms; Women's health.

\section{Resumen}

El objetivo de este estudio fue analizar la tendencia de las tasas de mortalidad por cáncer de mama en mujeres del estado de Santa Catarina, Brasil, entre 2000 y 2017. Se trata de un estudio ecológico realizado con datos de defunción 
del Sistema de Información de Mortalidad y datos poblacionales obtenidos de estimaciones proporcionadas por el Ministerio de Salud. En el análisis se utilizaron modelos de regresión lineal simple y polinomial. Entre 2000 y 2017 se registraron 7.363 muertes por cáncer de mama en mujeres de 20 años o más. Para el período en su conjunto, el 47,5\% del total de defunciones se produjo en el grupo de edad de 50 a 69 años $(\mathrm{n}=3.497)$. La tasa media de mortalidad por cáncer de mama fue de 18,24 / 100.000 mujeres (2000-2017). Los coeficientes medios de mortalidad superaron las 30 defunciones / 100.000 mujeres de 50 a 59 años, llegando a 87,25 / 100.000 en las más longevas. En el análisis de la tendencia, desagregando por grupos de edad, se observó, en general, un incremento lineal no constante en el período. El estudio reveló una tendencia creciente de mortalidad por cáncer de mama en mujeres residentes en el estado de Santa Catarina durante el período de estudio, y mostró la magnitud del aumento en los coeficientes medios según los grupos de edad considerados.

Palabras clave: Mortalidad aumentada; Epidemiología; Estudios de series temporales; Neoplasias de mama; La salud de la mujer.

\section{Introdução}

O câncer de mama é a neoplasia maligna mais prevalente e que provoca o maior número de óbitos por câncer na população feminina. No ano de 2018, o número de mortes foi estimado em 627.000, valor que corresponde a aproximadamente $15 \%$ de todas as mortes por câncer entre mulheres no mundo (WHO, 2020). No Brasil, a doença se configura como um problema de saúde pública importante, apresentando uma elevada incidência em mulheres de todas as regiões do país (INCA, 2019a; WHO, 2020), além de constituir a primeira causa de morte por câncer nas mulheres brasileiras (INCA, 2019a).

O câncer de mama é causado pela multiplicação desordenada das células que compõem o tecido mamário, originando células anormais que formarão um tumor. A doença pode progredir de formas distintas, a depender do tipo de câncer. Alguns são de rápida evolução, enquanto outros desenvolvem-se lentamente. É o tipo de câncer mais comum entre as mulheres, e embora possa também desenvolver-se na população masculina, os casos são considerados raros (INCA, 2019b).

A etiologia da doença é multifatorial, sendo que o câncer de mama se torna mais importante, em termos de frequência de ocorrência, no envelhecimento. Em adição ao processo de envelhecer, deve-se considerar a exposição de mulheres a fatores que aumentam o risco de surgimento da doença ao longo da vida, como os fatores genéticos ou hereditários, os relacionados à história hormonal e reprodutiva, além dos comportamentais e ambientais (Armstrong et al., 2019; INCA, 2019b). Idade avançada na primeira gestação, baixa paridade, períodos curtos de amamentação (comuns nas sociedades modernas), uso de álcool, obesidade e inatividade física são exemplos de fatores de risco importantes para o câncer de mama (Malta \& Silva Jr., 2014).

Destaca-se que a mortalidade por essa doença se distribui no país de forma heterogênea. Essa diferença pode estar relacionada aos fatores de risco para a doença, além de questões relacionadas ao desenvolvimento econômico, ao acesso aos serviços de saúde e às desigualdades sociais, nas distintas regiões do país (Duarte et al., 2020). Nas regiões Sul e Sudeste do Brasil observam-se as taxas de mortalidade mais elevadas, sendo que Santa Catarina (SC) está entre os sete estados brasileiros com as maiores incidências de câncer de mama feminino (INCA, 2019a). De acordo com as evidências apresentadas, surgiu a seguinte pergunta de pesquisa: os casos de óbitos por câncer de mama estão aumentando em Santa Catarina com o passar dos anos?

Conhecer os aspectos epidemiológicos relacionados à mortalidade por câncer de mama tornam-se úteis para a implementação de programas de controle e prevenção, além de favorecer o desenvolvimento de políticas públicas voltadas à promoção de saúde, que visem reduzir os impactos da doença na população feminina. Considerando a escassez de trabalhos que retratem a análise temporal dos óbitos por esse tipo de câncer em Santa Catarina, o presente estudo teve por objetivo analisar a tendência da mortalidade por câncer de mama no estado em um período de 17 anos. 


\section{Metodologia}

Trata-se de um estudo ecológico que analisou a tendência dos coeficientes de mortalidade por câncer de mama, de mulheres com idade $\geq 20$ anos, residentes no estado de Santa Catarina, no período compreendido entre os anos 2000 e 2017 .

\section{Fonte de dados}

Foram utilizados os registros de óbitos femininos por neoplasia maligna da mama - código C50 da Décima Revisão da Classificação Internacional de Doenças (CID-10) - durante o período proposto em Santa Catarina, tendo como referência o município de residência das mulheres, e disponibilizados pelo Sistema de Informação sobre Mortalidade (SIM) do Departamento de Informática do SUS (DATASUS). Os dados de óbitos foram agrupados nas seguintes faixas etárias: 20-39, 40-49, 50-59, 60-69, 70-79 e $\geq 80$ anos.

As projeções populacionais foram obtidas das estimativas preliminares elaboradas pelo Ministério da Saúde/Secretaria de Vigilância em Saúde (SVS)/Departamento de Análise em Saúde e Vigilância das Doenças Não Transmissíveis (DASNT)/Coordenação-Geral de Informações e Análises Epidemiológicas (CGIAE).

\section{Análise de dados}

Os dados gerados foram exportados para planilhas no Microsoft Excel® (versão 19), onde foi realizado o cálculo dos coeficientes anuais de mortalidade a cada ano, por 100 mil mulheres. Para a análise da tendência no período, utilizaram-se os coeficientes padronizados por idade para o conjunto de mulheres (método direto, considerando-se como população padrão brasileira do Censo Demográfico de 2010); e os coeficientes específicos para cada faixa etária.

Inicialmente observaram-se os diagramas de dispersão que mostraram o comportamento das taxas de mortalidade por câncer de mama nos anos de estudo. Em todos os casos observou-se que a suposição de uma evolução linear ou de um modelo polinomial (quadrático ou cúbico) poderia ser assumida (Moretin \& Toloi, 1986; Charnet et al., 2008). Em todas as séries relativas às faixas etárias consideradas, foi realizado alisamento por meio de médias móveis centrada em três termos.

Os coeficientes de mortalidade foram considerados como variável dependente (Y), e os anos calendários como variável independente $(\mathrm{X})$. Para atenuar a correlação serial de tempo, empregou-se a variável ano centrado (X - ponto médio da série histórica). Entre os modelos selecionados com base no coeficiente de determinação (R2), utilizou-se o mais parcimonioso (Charnet et al., 2008).

A verificação dos pressupostos de normalidade e homoscedasticidade foi realizada para garantir a validade das estimativas obtidas (Neter, Wasserman \& Kutner, 1990), por meio dos testes de Shapiro-Wilk (Shapiro \& Wilk, 1965), Breusch-Pagan-Godfrey (Godfrey, 1978), respectivamente. Considerou-se tendência significativa aquela cujo modelo estimado obteve $\mathrm{p}<0,05$. Para todos os testes estatísticos realizados neste estudo foi adotado um nível de significância de 5\%. As análises foram realizadas no Statistical Package for the Social Sciences (SPSS® versão 21). No presente estudo todos os dados utilizados são de domínio público.

\section{Resultados}

\section{Caracterização da amostra}

Durante o período proposto, foram identificados 7.363 óbitos por câncer de mama em mulheres com idade igual ou superior a 20 anos no estado de Santa Catarina. Na faixa etária compreendida entre 20 a 39 anos ocorreram 566 óbitos (7,7\%), de 40 a 49 anos 1.356 óbitos $(18,4 \%$ ), sendo que 47,5\% do total de óbitos ocorreu na faixa etária de 50 a 69 anos (n=3.497). Na faixa de 70 a 79 anos ocorreram 1.120 óbitos (15,2\%), e a partir dos 80 anos detectaram-se 824 óbitos (11,2\%). 


\section{Resultados obtidos}

No que se refere à distribuição proporcional dos óbitos de acordo com o ano para o conjunto das mulheres adultas, a mortalidade por câncer de mama passou de 3,78\% em 2000, para 8,38\% no ano de 2017. A tendência dos coeficientes padronizados de mortalidade por câncer de mama para as mulheres no estado de Santa Catarina é apresentada na Figura 1. O coeficiente médio no período foi de 18,24 óbitos/100.000 mulheres com aumento linear não constante no período (Tabela 1).

Figura 1 - Tendência dos coeficientes padronizados de mortalidade por câncer de mama (por 100.000 mulheres), estado de Santa Catarina, 2000 a 2017.

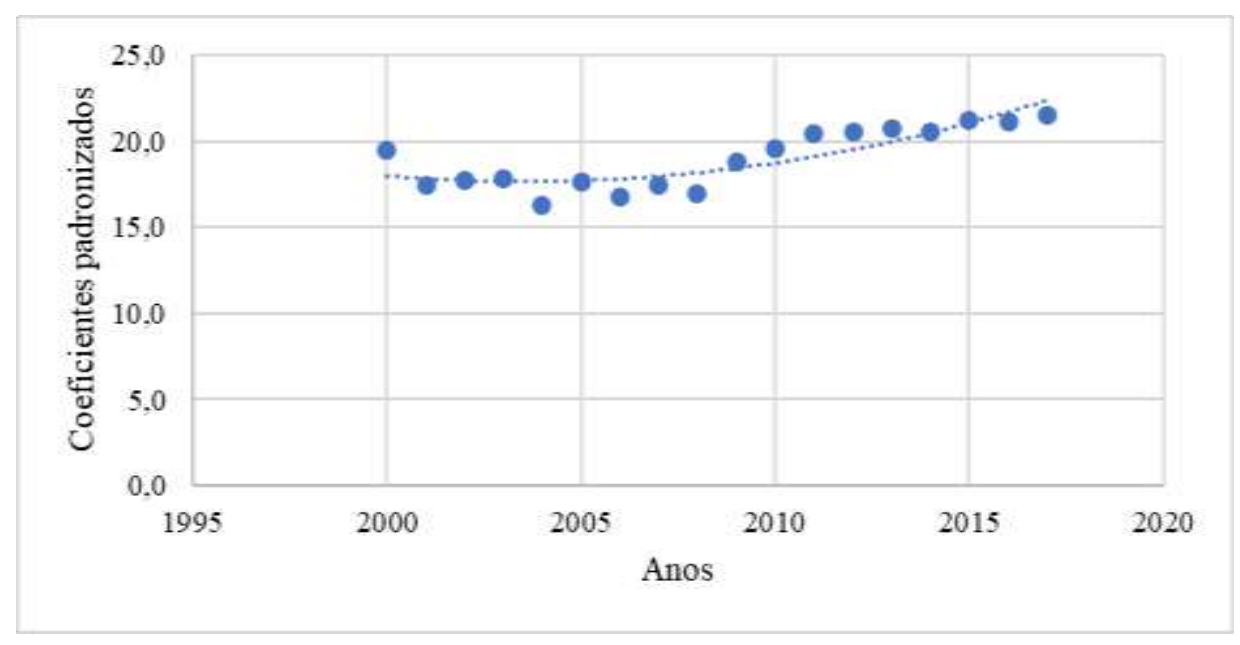

Fonte: Autores.

$\mathrm{Na}$ análise das tendências segundo faixas etárias, observou-se redução constante dos coeficientes nas faixas etárias de 20 a 39 anos, 60 a 69 anos, e 70 a 79 anos no período estudado ( p < 0,0001). Porém, com o aumento da idade, observa-se elevação crescente dos coeficientes de mortalidade, sendo que na faixa de 50 a 59 atinge quase o dobro do observado para o grupo de 40 e 49 anos, e chega a 87,25/100.000 mulheres com idade igual ou maior a 80 anos (Tabela 1).

Tabela 1 - Coeficientes de regressão e significância estatística da tendência da mortalidade por câncer de mama, segundo faixas etárias, estado de Santa Catarina, 2000 a 2017.

\begin{tabular}{ccccccc}
\hline Faixas etárias & $\beta_{0}$ & $\beta_{1}$ & $\beta_{2}$ & $\beta_{3}$ & $\mathrm{p}(\mathrm{F})$ & $\mathrm{R}^{2}$ \\
\hline 20 a $39^{\mathrm{a}}$ & 3,00 & $-0,09$ & & & $<0,001$ & 0,60 \\
40 a $49^{\mathrm{b}}$ & 16,55 & $-0,46$ & 0,09 & & $<0,001$ & 0,72 \\
50 a $59^{\mathrm{c}}$ & 30,55 & $-0,59$ & 0,27 & & $<0,001$ & 0,77 \\
60 a $69^{\mathrm{d}}$ & 43,21 & $-1,47$ & & & $<0,001$ & 0,81 \\
70 a $79^{\mathrm{e}}$ & 54,90 & $-2,61$ & & & $<0,001$ & 0,71 \\
80 anos e mais $^{\mathrm{f}}$ & 87,25 & $-6,82$ & 0,72 & 0,40 & 0,005 & 0,65 \\
Total $^{\mathrm{g}}$ & 18,24 & $-0,49$ & 0,09 & & $<0,001$ & 0,79 \\
\hline
\end{tabular}

Nota: a, b, c, d, e, f - séries alisadas; g - série original. Fonte: Autores.

\section{Discussão}

A partir dos resultados encontrados, foi possível verificar que há tendência crescente de mortalidade por câncer de mama em mulheres residentes em Santa Catarina no período estudado. O aumento observado é expressivo, de 3,78\% para 
8,38\%, podendo ser explicado por vários fatores como o crescimento populacional, aprimoramento da notificação dos dados de mortalidade e diminuição da mortalidade por causas mal definidas (França et al., 2014). Além disso, são fatores relacionados: as mudanças no estilo de vida, resultantes obesidade em mulheres na pós-menopausa, maior consumo de álcool, inatividade física e utilização de terapia de reposição hormonal por longos períodos (Youlden et al., 2012; Neuhouser et al., 2015). Nos últimos anos, com as mudanças na expectativa de vida e alteração da pirâmide etária, observa-se um maior número de mulheres idosas, o que também predispõe a população feminina ao câncer de mama (Meira et al., 2015).

Santa Catarina é o estado que apresenta a maior expectativa de vida do Brasil (IBGE, 2018). De acordo com as informações do último censo demográfico (2010), a população feminina acima de 65 anos no estado era de 245.221 mulheres. A população é composta por 6.248.436 habitantes, dos quais 3.148.076 são mulheres (IBGE, 2021). De acordo com as estimativas, até o ano de 2022, 3.370 mulheres catarinenses poderão ser acometidas pelo câncer de mama (INCA, 2019a). No ano de 2020, os óbitos femininos por neoplasias malignas ocorridos no estado foram relacionados em maior parte às neoplasias mamárias (35,1\%) (DIVE, 2020).

A partir do ano 2000, ocorreu uma queda discreta nos coeficientes de mortalidade, mas após o ano de 2008, a mortalidade aumentou gradativamente. Essa queda inicial está de acordo com o observado para a região Sul do Brasil, período em que as taxas de mortalidade diminuíram (Couto, 2016). Com o passar dos anos, a mortalidade por câncer de mama continua aumentando, seguindo a tendência apresentada pelo país (Barros, 2020). Outros estados brasileiros também apresentam tendência crescente de mortalidade, a exemplo de Alagoas (Lôbo et al., 2020), Ceará (Barros, 2020) e Bahia (Souza et al., 2020).

Quanto ao comportamento da distribuição da mortalidade por câncer de mama, de acordo com as faixas etárias avaliadas neste estudo, verificou-se uma relação positiva com o avançar da idade, destacando-se as faixas etárias a partir de 50 a 59 anos. O coeficiente de mortalidade para mulheres de 50 a 59 anos quase dobrou em relação à faixa etária de 40 a 49 anos, com aumentos constantes nas sucessivas faixas etárias até os 79 anos. No estado de Alagoas, no período de 2001 a 2016, a mortalidade também cresceu acompanhando o aumento da faixa etária, denotando que o envelhecimento é um fator importante na casuística do câncer de mama (Lôbo et al., 2020).

No Brasil, considerando toda a sua extensão geográfica, o aumento da mortalidade por câncer de mama após os 50 anos de idade pode ser observado, assim como a detecção de maiores taxas de mortalidade na faixa etária superior aos 75 anos (Meira et al., 2015), de forma semelhante aos resultados evidenciados neste estudo. Assim, ressalta-se a necessidade de detecção precoce do câncer de mama.

Além do exame clínico, o rastreamento mamográfico possui posição de destaque entre as medidas de prevenção, diagnóstico precoce, controle do câncer e diminuição dos índices de mortalidade no país. Algumas pesquisas retratam altas taxas de óbitos por câncer de mama (85-90\%) em mulheres com idade superior a 40 anos (Souza et al., 2017; Barros et al., 2020). No entanto, no serviço público de saúde brasileiro, a mamografia é realizada somente a partir dos 50 anos, a cada dois anos até os 69 anos (INCA, 2019c), faixa etária considerada de risco.

O rastreamento mamográfico na faixa etária dos 40 anos, assim como acima de 70 anos, tem repercutido em amplas discussões no país. Visto que a incidência de câncer de mama no Brasil, na faixa etária de 40 a 50 anos é maior do que a de países desenvolvidos, e que as taxas de câncer de mama nessa faixa representam 74\% dos casos (Dibaba et al., 2018), a Sociedade Brasileira de Mastologia sugere a inclusão desse grupo etário para a detecção do câncer em seus estágios ainda iniciais, por meio do rastreamento (Urban et al., 2017; SBM, 2021). Porém, discute-se sobre as possibilidades de ocorrência de sobrediagnóstico, quando tumores de comportamento indolente são identificados na mamografia. Não representam ameaça à vida, mas podem gerar, consequentemente, sobretratamentos, como procedimentos cirúrgicos e tratamentos oncológicos desnecessários, a exemplo da radioterapia e quimioterapia (Gøtzsche, 2013). 
Alguns autores afirmam que a deteção precoce do câncer de mama, em estágios menos avançados, pode proporcionar uma sobrevida maior ao paciente, com maiores chances de cura (Hofelmann, Anjos \& Ayala, 2014). De acordo com o estudo realizado por Rosa et al. (2016), o rastreamento mamográfico no Brasil e em Santa Catarina (2009 a 2012), para a faixa de 45 a 49 anos, ocorreu em apenas $22,2 \%$ e 23,1\% das mulheres, respectivamente. Mesmo com o baixo percentual de realização do exame, verificaram que realizar a mamografia a partir dos 40 anos contribuiu para o diagnóstico do câncer de mama entre os 40 e 49 anos. Nesse mesmo estudo, foi apontado um índice de aproximadamente $42 \%$ referente a realização de exames mamográficos em Santa Catarina no ano de 2012, para a faixa de 50 a 69 anos, muito abaixo do preconizado pela Organização Mundial da Saúde (70\%) (Rosa et al., 2016).

De acordo com informações do Ministério da Saúde, em pesquisa realizada nas capitais brasileiras sobre a vigilância de fatores de risco para as doenças crônicas, aproximadamente 86\% das mulheres de 50 a 69 anos, residentes em Florianópolis, capital catarinense, realizaram o rastreamento mamográfico em 2017 (Ministério da Saúde, 2018). Embora este índice de cobertura seja bom, pode não refletir o contexto de disparidades existentes nos outros municípios do estado.

Ressalta-se que em Santa Catarina muitos municípios são predominantemente rurais, característica que pode não favorecer o acesso aos serviços de saúde, o que necessita de maiores investigações (Barbosa et al., 2015). Nas regiões distintas do país ocorrem variações no acesso aos serviços de saúde e à qualidade da assistência prestada às mulheres com câncer, assim como nas capitais e cidades no interior dos estados, o que pode refletir nas taxas de mortalidade por câncer de mama (Gonçalves et al., 2014).

Em Santa Catarina, que se destaca no setor de produção agrícola e animal, um fator importante a ser avaliado é a exposição ambiental aos agrotóxicos. O uso intensivo dessas substâncias é um problema de saúde pública no país, podendo haver relação entre áreas com maior consumo de agrotóxicos disruptores endócrinos e o aumento das taxas de mortalidade por cânceres como o de mama (Dutra et al., 2021).

O coeficiente médio de mortalidade por câncer de mama, para o período estudado, foi de 18,24 óbitos/100.000 mulheres. A magnitude deste coeficiente deve ser considerada, visto que o Brasil, de 1980 a 2016, apresentou taxas padronizadas de mortalidade inferiores, que variaram de 9,2 a 12,4 por 100 mil mulheres. Embora a taxa de mortalidade do Brasil aumente, observam-se diferenças regionais, sendo que Norte e Nordeste possuem taxas de mortalidade por câncer de mama menores do que as regiões Sul, Sudeste e Centro-oeste (Dibaba et al., 2018).

Nas regiões com maior incidência e, consequentemente, maior mortalidade, fatores relacionados ao estilo de vida e cultura alimentar podem estar associados ao câncer de mama. Sabe-se que hábitos alimentares inadequados levam a obesidade. Na pós-menopausa, o risco de câncer aumenta conforme se elevam o índice de massa corporal e a adiposidade abdominal nas mulheres, podendo refletir em um número maior de casos de câncer de mama (Neuhouser et al., 2015).

No presente estudo, a mortalidade na faixa etária de 80 anos ou mais foi $30 \%$ maior que a observada em comparação as outras faixas de idade, corroborando outros estudos nacionais (Costa et al., 2019; Lôbo et al., 2020; Souza et al., 2020). De fato, o envelhecimento segue como um dos fatores de risco mais relevantes para o aumento da incidência e mortalidade por câncer de mama (Barros et al., 2020). Em decorrência do processo fisiológico do envelhecimento, naturalmente os sistemas imunológico e neuroendócrino entram em declínio, havendo maior susceptibilidade para o desenvolvimento de infecções, alterações hormonais e eventos carcinogênicos (Macena, Hermano \& Costa, 2018).

Além disso, o tratamento oncológico torna-se mais agressivo para pessoas em idades avançadas, elevando o risco de óbito, motivo pelo qual muitas mulheres diagnosticadas com câncer nessas faixas etárias optam pelo curso da história natural da doença (Souza et al., 2015). Deve-se considerar também que na faixa etária $\geq 80$ anos, cuja expectativa de vida foi superada, poderá haver maior mortalidade, independentemente do diagnóstico de câncer, devido à alta prevalência de multimorbidade em idosas longevas (Leite et al., 2020). 
Importante salientar que mulheres idosas acima de 70 anos não possuem recomendação para realizar a mamografia bienal, conforme as orientações do Ministério da Saúde (Souza et al., 2020). Assim, essa população participa em menor proporção dos programas de rastreamento, o que pode predispor a prejuízos na sobrevida (Pinheiro, 2015).

Como a etiologia do câncer é multifatorial, deve-se considerar os fatores individuais, que incluem o estilo de vida, a alimentação, a atividade física e a constituição genética, assim como fatores ambientais e socioeconômicos, que também interferem nessa problemática (INCA, 2019b). Na perspectiva da epidemiologia socioambiental, frente a complexidade dos elementos que influenciam na saúde dos indivíduos, é importante verificar a associação entre as condições do ambiente e a vulnerabilidade a determinados tipos de doenças (Alves \& Ribeiro Filho, 2020), sobretudo na população idosa.

Atualmente, frente aos resultados apresentados por este estudo, considerando a diversidade de fatores determinantes para o câncer de mama, torna-se um desafio a adequação dos serviços de saúde às ações necessárias para ampliar as medidas de prevenção. Deve-se refletir sobre a necessidade de intensificar as políticas de adesão ao rastreamento mamográfico, de incluir as mulheres com idade superior a 40 anos ao rastreio, e monitorar o acesso das mulheres mais idosas aos serviços de saúde.

Devido ao impacto da pandemia de COVID-19 sobre a saúde pública e economia do Brasil, nos anos 2020 e 2021, o desafio quanto às medidas de prevenção será ainda maior, em decorrência de interrupções no rastreamento. $\mathrm{O}$ controle precoce do câncer é uma preocupação durante a pandemia, visto que a falta de acesso oportuno aos serviços pode impactar no tratamento e sobrevivência das mulheres acometidas pelo câncer de mama (Figueroa et al., 2021).

O conhecimento do perfil de óbitos em Santa Catarina, além de contribuir para a identificação dos grupos de maior risco, pode subsidiar ações que favoreçam a prevenção, o diagnóstico precoce, a cura ou a melhoria na qualidade de vida das mulheres com câncer, além da redução dos gastos públicos. O presente estudo apresenta as limitações próprias do delineamento (ecológico) empregado e do uso de dados secundários de registros de óbitos e estimativas populacionais.

\section{Conclusão}

A análise realizada neste estudo evidenciou uma tendência crescente de mortalidade por câncer de mama em mulheres residentes no estado de Santa Catarina no período estudado. A mortalidade se elevou de acordo com o avançar da idade, havendo predomínio nas mais longevas. O coeficiente médio no período esteve acima daquele observado para o Brasil para essa faixa etária, apontando assim a necessidade de monitoramento dessa doença no estado, buscando-se identificar possíveis relações entre fatores socioambientais e a incidência do câncer de mama em Santa Catarina em estudos futuros.

\section{Referências}

Alves, L. A., \& Ribeiro Filho, V. (2020). Usos do solo e ocorrências de câncer: uma abordagem geográfica a partir de São Gotardo (MG). Espaço em Revista, 22 (2), 56-78. https://www.revistas.ufg.br/espaco/article/view/63687

Armstrong, N., Ryder, S., Forbes, C., Ross, J., \& Quek, R. G. (2019). A systematic review of the international prevalence of BRCA mutation in breast cancer. Clinical Epidemiology, 11, 543-561. https://www.ncbi.nlm.nih.gov/pmc/articles/PMC6628947/

Barbosa, I. R., Costa, I. D. C. C., Pérez, M. M. B., \& de Souza, D. L. B. (2015). As iniquidades sociais e as disparidades na mortalidade por câncer relativo ao gênero. Revista Ciência Plural, 1 (2), 79-86. https://periodicos.ufrn.br/rcp/article/view/7618

Barros, L. O., Menezes, V. B. B., Jorge, A. C., de Morais, S. S. F., \& da Silva, M. G. C. (2020). Mortalidade por Câncer de Mama: uma Análise da Tendência no Ceará, Nordeste e Brasil de 2005 a 2015. Revista Brasileira de Cancerologia, 66 (1), 1-8. https://rbc.inca.gov.br/revista/index.php/revista/article/view/740

Charnet, R., Freire, C. A. L., Charnet, E. M. R., \& Bonvino, H. (2008). Análise de modelos de regressão linear: com aplicações. Campinas: Editora da Unicamp.

Costa, L. D. L. N., de Lima Sardinha, A. H., Verzaro, P. M., Lisbôa, L. L. C., \& Batista, R. F. L. (2019). Mortalidade por câncer de mama e condições de desenvolvimento humano no Brasil. Revista Brasileira de Cancerologia, 65 (1), e-12050. https://rbc.inca.gov.br/revista/index.php/revista/article/view/50

Couto, M. S. A. (2016). Análise da taxa de mortalidade por câncer de mama nos municípios brasileiros no período de 1987 a 2013 e fatores associados [dissertação]. Juiz de Fora, MG: Universidade Federal de Juiz de Fora. 
Dibaba, D. T., Ogunsina, K., Braithwaite, D., \& Akinyemiju, T. (2019). Metabolic syndrome and risk of breast cancer mortality by menopause, obesity, and subtype. Breast Cancer Research and Treatment, 174 (1), 209-218. https://link.springer.com/article/10.1007/s10549-018-5056-8

Diretoria de Vigilância Epidemiológica. (2020). Barriga Verde Informativo Epidemiológico. Ano XV- Edição Especial. Santa Catarina. http://dive.sc.gov.br/barrigaverde/pdf/BV_Cancer_27.11.pdf.

Duarte, D. D. A. P., Nogueira, M. C., Magalhães, M. D. C., \& Bustamante-Teixeira, M. T. (2020). Iniquidade social e câncer de mama feminino: análise da mortalidade. Cadernos Saúde Coletiva, 28, 465-476. https://www.scielo.br/j/cadsc/a/7KtMNqFxJZSPGYRB3FzgsZj/?format=html\&stop=previous\&lang=pt

Dutra, L. S., Ferreira, A. P., Horta, M. A. P., \& Palhares, P. R. (2021). Uso de agrotóxicos e mortalidade por câncer em regiões de monoculturas. Saúde em Debate, 44, 1018-1035. https://www.scielo.br/j/sdeb/a/FfpPSnKCkxrdqPd8ptnfWsJ/?format=html\&lang=pt

França, E., Teixeira, R., Ishitani, L., Duncan, B. B., Cortez-Escalante, J. J., Morais Neto, O. L. D., \& Szwarcwald, C. L. (2014). Causas mal definidas de óbito no Brasil: método de redistribuição baseado na investigação do óbito. Revista de Saúde Pública, 48, 671-681. https://www.scielosp.org/article/rsp/2014.v48n4/671-681/pt/

Figueroa, J. D., Gray, E., Pashayan, N., Deandrea, S., Karch, A., Vale, D. B., \& Breast Screening Working Group. (2021). The impact of the Covid-19 pandemic on breast cancer early detection and screening. Preventive Medicine, 151, 106585. https://www.sciencedirect.com/science/article/pii/S0091743521001699

Godfrey, L. G. (1978). Testing against general autoregressive and moving average error models when the regressors include lagged dependent variables. Econometrica: Journal of the Econometric Society, 46, 1293-1301. https://www.jstor.org/stable/1913829

Gonçalves, L. L. C., Travassos, G. L., Almeida, A. M. D., Guimarães, A. M. D., \& Gois, C. F. L. (2014). Barreiras na atenção em saúde ao câncer de mama: percepção de mulheres. Revista da Escola de Enfermagem da USP, 48, 394-400. https://www.scielo.br/j/reeusp/a/9RfKfjrRGXmWNxvMYLSZdXD/abstract/?lang=pt

Gøtzsche, P. C., \& Jørgensen, K. J. (2013). Screening for breast cancer with mammography. Cochrane Database of Systematic Reviews, (6). https://www.cochranelibrary.com/cdsr/doi/10.1002/14651858.CD001877.pub5/full

Höfelmann, D. A., Anjos, J. C. D., \& Ayala, A. L. (2014). Sobrevida em dez anos e fatores prognósticos em mulheres com câncer de mama em Joinville, Santa Catarina, Brasil. Ciência \& Saúde Coletiva, 19, 1813-1824. https://www.scielo.br/j/csc/a/TKzFdJ4MywrWDW6SmQLb66n/abstract/?lang=pt

Instituto Brasileiro de Geografia e Estatística (2018). Agência IBGE notícias. Expectativa de vida do brasileiro sobe para 75,8 anos. https://agenciadenoticias.ibge.gov.br/agencia-noticias/2012-agencia-de-noticias/noticias/18469-expectativa-de-vida-do-brasileiro-sobe-para-75-8-anos

Instituto Brasileiro de Geografia e Estatística. (2021). Sinopse do censo demográfico 2010 . Santa Catarina. https://censo2010.ibge.gov.br/sinopse/index.php?uf=42\&dados=26.

Instituto Nacional de Câncer José Alencar Gomes da Silva. (2019a). Estimativa 2020: incidência de câncer no Brasil. https://www.inca.gov.br/sites/ufu.sti.inca.local/files//media/document//estimativa-2020-incidencia-de-cancer-no-brasil.pdf

Instituto Nacional de Câncer José Alencar Gomes da Silva. (2019b). Tipos de câncer. Câncer de mama. https://www.inca.gov.br/tipos-de-cancer

Instituto Nacional de Câncer José Alencar Gomes da Silva. (2019c). A situação do câncer de mama no Brasil: síntese de dados dos sistemas de informação. https://www.inca.gov.br/publicacoes/livros/situacao-do-cancer-de-mama-no-brasil-sintese-de-dados-dos-sistemas-de-informacao.

Leite, B. C., Oliveira-Figueiredo, D. S. T. D., Rocha, F. L., \& Nogueira, M. F. (2020). Multimorbidade por doenças crônicas não transmissíveis em idosos: estudo de base populacional. Revista Brasileira de Geriatria e Gerontologia, 22 (6), e190253. https://www.scielo.br/j/rbgg/a/yPJDvn3XN5wbTBp6Scjq9Pz/?lang=pt

Lôbo, J. L. D. S., Silva, M. L. C., Tomé, T. K. B. V., \& Souza, C. D. F. D. (2020). Mortalidade por Câncer de Mama Feminino em Alagoas no Período de 2001 a 2016: Análise de Tendência e Distribuição Espacial. Revista Brasileira de Cancerologia, $66 \quad$ (1), 1-7. https://pesquisa.bvsalud.org/controlecancer/resource/pt/biblio-1094926?src=similardocs

Macena, W. G., Hermano, L. O., \& Costa, T. C. (2018). Alterações fisiológicas decorrentes do envelhecimento. Revista Mosaicum, 15 (27), $223-238$. https://revistamosaicum.org/index.php/mosaicum/article/view/64

Malta, D. C., \& Silva Jr, J. B (2014). Plano de ações estratégicas para o enfrentamento das doenças crônicas não transmissíveis no Brasil após três anos de implantação, 2011-2013. $\quad$ Epidemiologia $\quad$ e $\quad$ Serviços $\quad$ de $\quad$ Saúde; $\quad 23 \quad 389-95$. https://www.scielo.br/j/ress/a/WSszv8nc9DTwPpR9YMG59pm/?format=pdf\&lang=pt

Meira, K. C., Guimarães, R. M., Santos, J. D., \& Cabrelli, R. (2015). Análise de efeito idade-período-coorte na mortalidade por câncer de mama no Brasil e regiões. Revista Panamericana de Salud Pública, 437 (6), https://www.scielosp.org/article/ssm/content/raw/?resource_ssm_path=/media/assets/rpsp/v37n6/v37n6a05.pdf

Ministério da Saúde. (2018). Vigitel Brasil 2017: vigilância de fatores de risco e proteção para doenças crônicas por inquérito telefônico: estimativas sobre frequência e distribuição sociodemográfica de fatores de risco e proteção para doenças crônicas nas capitais dos 26 estados brasileiros e no Distrito Federal em 2017. https://bvsms.saude.gov.br/bvs/publicacoes/vigitel_brasil_2017_vigilancia_fatores_riscos.pdf

Moretin, P. A., \& Toloi, C. M. (1986). Séries temporais. São Paulo: Atual.

Neter, J., Wasserman, W., \& Kutner, M. H. (1990). Polynomial regression. In: Neter, J., Wasserman, W., Kutner, M. H., editors. Applied linear statistical models: regression, analysis of variance and experimental designs. Ed. Boston. 315-41. 
Neuhouser, M. L., Aragaki, A. K., Prentice, R. L., Manson, J. E., Chlebowski, R., Carty, C. L., \& Anderson, G. L. (2015). Overweight, obesity, and postmenopausal invasive breast cancer risk: a secondary analysis of the women's health initiative randomized clinical trials. JAMA Oncology, 1 (5), 611-621. https://jamanetwork.com/journals/jamaoncology/article-abstract/2319235

Pinheiro, S. J. (2015). Câncer de mama: análise da mortalidade e do cuidado de enfermagem no município de Fortaleza - Ceará [dissertação]. Fortaleza (CE): Universidade Estadual do Ceará. https://bdtd.ibict.br/vufind/Record/UECE-0_0745bfc3fc41c803959d6b4919aadc0f

Rosa, L. M. D., Silva, L. D., Radunz, V., \& Arzuaga, M. A. (2016). Rastreamento mamográfico: detecção de lesões neoplásicas malignas em mulheres de Santa Catarina e do Brasil. Texto \& 25 Contexto-Enfermagem, (3), https://www.scielo.br/j/tce/a/RfnRQgZnrm8ZXrSZsq6xBBm/?lang=pt\&format=html

Shapiro, S. S., \& Wilk, M. B. (1965). An analysis of variance test for normality (complete samples). Biometrika, 52 (3/4), 591-611. https://www.jstor.org/stable/2333709

Sociedade Brasileira de Mastologia. (2021). Sociedades brasileiras recomendam mamografia a partir dos 40 anos. https://sbmastologia.com.br/sociedadesmedicas-brasileiras-recomendam-mamografia-anual-a-partir-dos-40-anos/

Souza, C. B., Fustinoni, S. M., Amorim, M. H. C., Zandonade, E., Matos, J. C., \& Schirmer, J. (2015). Estudo do tempo entre o diagnóstico e início do tratamento do câncer de mama em idosas de um hospital de referência em São Paulo, Brasil. Ciência \& Saúde Coletiva, 20 (12), $3805-3816$. https://www.scielo.br/j/csc/a/GnqNTpPc7pxpDNN37tR3Pbt/abstract/?lang=pt

Souza, K. S., da Silva, M. R. F., dos Santos Silva, R. R., dos Santos Silva, J., de Oliveira, M. B. M., Miranda, F. S. L., \& Lima, F. L. O. (2020). Tendência de mortalidade por Câncer de Mama na Bahia: 2008-2018. Brazilian Journal of Health Review, 3 (5), 14521-14528. https://www.brazilianjournals.com/index.php/BJHR/article/view/18299

de Souza, N. H. A., Falcão, L. M. N., Nour, G. F. A., Brito, J. O., Castro, M. M., \& de Oliveira, M. S. (2017). Câncer de mama em mulheres jovens: estudo epidemiológico no Nordeste brasileiro. SANARE-Revista de Políticas Públicas, 16 (2), 60-67. https://sanare.emnuvens.com.br/sanare/article/view/1179

Urban, L. A. B. D., Chala, L. F., Bauab, S. D. P., Schaefer, M. B., Santos, R. P. D., Maranhão, N. M. D. A., \& Camargo, H. S. A. D. (2017). Recomendações do Colégio Brasileiro de Radiologia e Diagnóstico por Imagem, da Sociedade Brasileira de Mastologia e da Federação Brasileira das Associações de Ginecologia e Obstetrícia para o rastreamento do câncer de mama. Radiologia Brasileira, 50 (4), 244-249. https://www.scielo.br/j/rb/a/mdrskx4dDvTfVZxqkFp8ZKr/abstract/?lang=pt

World Health Organization (2020). Breast cancer. https://www.who.int/cancer/prevention/diagnosis-screening/breast-cancer/en/cited

Youlden, D. R., Cramb, S. M., Dunn, N. A., Muller, J. M., Pyke, C. M., \& Baade, P. D. (2012). The descriptive epidemiology of female breast cancer: an international comparison of screening, incidence, survival and mortality. Cancer Epidemiology, 36 (3), 237-248. https://www.sciencedirect.com/science/article/abs/pii/S187778211200029X 